\title{
ANÁLISE COMPARATIVA DO SISTEMA DE INFORMAÇÃO DE MORTALIDADE ENTRE MUNICÍPIOS DE UMA REGIONAL DE SAÚDE DO ESTADO DE PERNAMBUCO
}

\section{COMPARATIVE ANALYSIS ON THE MORTALITY INFORMATION SYSTEM BETWEEN MUNICIPALITIES OF A REGIONAL HEALTH OF THE STATE OF PERNAMBUCO}

\author{
Jonnathan Anderson da Silva Santos ${ }^{1}$ \\ Daniele Ferreira Rodrigues ${ }^{2}$
}

RESUMO

Objetivo: Comparar a qualidade da informação do Sistema de Informação sobre Mortalidade (SIM) nos municípios que compõe a VI Região de Saúde de Pernambuco no ano 2013. Métodos: Estudo comparativo com dados secundários do SIM avaliou a completitude das variáveis: raça/cor, estado civil, escolaridade e ocupação e o preenchimento da causa básica de óbito. Foram calculados os coeficientes de mortalidade por causas mal definidas por município e os percentuais de não preenchimento das variáveis, aplicou-se um escore de classificação entre muito ruim e excelente. Resultados: Foi identificada uma mortalidade de 4 óbitos por 1000 habitantes na VI Região de Saúde, sendo a maior em Arcoverde (9,1/1000 hab). Em relação à completitude observaram-se diferenças entre os municípios, tendo Inajá, Pedra, Sertânia e Venturosa apresentado índices mais elevados de completitude dos dados. Para a Região de Saúde as variáveis foram classificadas como padrão de qualidade bom (raça-cor), regular (estado civil e regularidade) e ruim (ocupação). O percentual de óbitos por causas mal definidas variou de $3,66 \%$ em Venturosa a $52,08 \%$ em Buíque. Conclusão: Observou-se grande variabilidade nas informações sobre mortalidade entre os municípios avaliados. A incompletude e o elevado índice de mortes por causas mal definidas refletem a qualidade do preenchimento e processamento das declarações de óbito e, consequentemente, a qualidade do SIM.

DESCRITORES: Sistemas de Informação em Saúde. Atestado de Óbito. Mortalidade. Causa de óbito. Epidemiologia Descritiva. Classificação Internacional de Doenças.

\begin{abstract}
Objective: To compare the quality of information from the Mortality Information System (SIM) in the municipalities that make up the VI Health Region of Pernambuco in the year 2013. Methods: Comparative study with SIM secondary data, evaluated the completeness of variables: race/color, marital status, education and occupation and filling the basic cause of death. Mortality rates by undefined causes were calculated by the municipality and the percentage of non-fulfillment of the variables, we applied a rating score between very bad and excellent. Results: The mortality of 4 deaths per 1000 inhabitants in the VI Health Region has been identified, the highest being in Arcoverde (9.1 / 1000 inhab.). Regarding the completeness, it was observed differences between the municipalities, and Inajá, Pedra, Sertânia and Venturosa presented higher rates of completeness of the data. Health Region variables were classified as standard good quality (race-color), regular (marital status and regularity) and bad (Occupation). The percentage of Mortality rates by undefined causes ranged from $3.66 \%$ in Venturosa to $52.08 \%$ in Buíque. Conclusion: There was great variability in the quality of information on mortality among municipalities assessed. The incompleteness and the high rate of deaths from undefined causes reflect the quality of information and processing of death certificates and hence the quality of the SIM.
\end{abstract}

DESCRIPTORES: Health Information Systems. Death Certificates. Mortality. Cause of Death. Descriptive Epidemiology. International Classification of Diseases.

1 Escola de Governo em Saúde Pública de Pernambuco - ESPPE / Universidade de Pernambuco - UPE.

2 Universidade de Pernambuco - UPE/ Residência Multiprofissional em Saúde Coletiva com Ênfase em Gestão de Redes de Atenção à Saúde da Universidade de Pernambuco. 
s Sistemas de Informação em Saúde (SIS) constituem-se de peças articuladas que tem por objetivo selecionar dados e produzir informações, auxiliar na formulação e avaliação de políticas e no processo de tomada de decisão, visando a melhoria da saúde da população'. Com a concepção do SUS e a promulgação da Lei $n^{\circ}$. 8.080, de 19 de setembro de1990, foi reforçada a importância do SIS para o sistema de saúde, tornando-o peça fundamental para a análise da situação de saúde de determinado local e população, subsidiando o planejamento das ações em saúde ${ }^{2}$.

Uma das principais fontes de informações sobre mortalidade no Brasil é o Sistema de Informação sobre Mortalidade (SIM) ${ }^{3-5}$. Este sistema é considerado uma importante fonte de informação para as estatísticas vitais, por meio dele, torna-se possível o monitoramento do estado de saúde das populações, identificação de grupos de risco, planejamento, definição e implantação de políticas públicas ${ }^{6}$.

A implantação do SIM representou um grande avanço na produção de informações sobre mortalidade no país. Foi implantado em 1975, com o objetivo de fornecer informações sobre a mortalidade para diversas instâncias, onde todo e qualquer óbito ocorrido no território nacional deve ser notificado por meio da Declaração de Óbito (DO $)^{7-3}$. Com a utilização de um formulário padronizado e individualizado para o registro dos dados, a DO, houve a possibilidade de se obter, para todo o território nacional, estatísticas de meIhor qualidade e com a grande vantagem de serem comparadas entre as regiões e entre outros países ${ }^{8}$.

A DO é composta por nove blocos:
I - Bloco de identificação do cartório que irá expedir o Atestado de Óbito; II: Bloco de identificação do indivíduo; III: Bloco de Residência do indivíduo; IV: Bloco de Ocorrência do óbito; V: Bloco destinado aos óbitos fetais ou em menores de um ano; VI: Bloco sobre as condições e causas do óbito; VII: Bloco de identificação do médico que preencheu a DO; VIII: Bloco destinado às causas externas e IX: Bloco destinado às localidades sem médico ${ }^{9}$. A DO é impressa em sequência numérica única e possui três vias coloridas ( $1^{\mathrm{a}}$ via Branca, $2^{a}$ via - Amarela e a $3^{a}$ via - Rosa) e autocopiativas.

Grande parte das informações presentes no SIM vem diretamente das Declarações de Óbitos (DO), contudo também são realizadas buscas ativas de dados em âmbito municipal, não se limitando, portanto, aos dados fornecidos pelo médico durante o preenchimento da DO. O processamento desses dados e a alimentação das bases de dados nacional são de responsabilidade das Secretarias Municipais de Saúde (SMS) e ocorrem atualmente via internet, com periodicidade regulamentada em portarias ministeriais. $O$ Ministério da Saúde (MS) é responsável por consolidar e disponibilizar esses dados no Departamento de Informática do Sistema Único de Saúde - DATASUS ${ }^{10}$.

As informações obtidas a partir das notificações são de grande importância, pois possibilitam a monitorização espaço-temporal de epidemias no país, bem como o acompanhamento da disseminação de doenças por categoria de exposição, subsidiando as ações para sua prevenção e controle ${ }^{11}$, principalmente em nível regional, onde o planejamento de ações e a organização de serviços de saúde de acordo com as necessidades da população 
se tornam mais específicos e muitas vezes mais resolutivos. Contudo, para que essas informações sejam precisas e oportunas é necessário que as várias etapas do sistema funcionem de forma articulada ${ }^{12}$.

Apesar da existência de dispositivos legais quanto à obrigatoriedade do registro do óbito, ainda existem muitas falhas relacionadas à cobertura do sistema de informação e ao preenchimento da DO. Cobertura e confiabilidade são características essenciais que conferem credibilidade ao sistema, estas características, entretanto, ficam comprometidas pela subnotificação de eventos e pelo preenchimento incorreto e/ou incompleto das DO.

As avaliações da completitude das variáveis dos principais campos do SIM envolvem a fidedignidade e consistências das informações e sinalizam a qualidade das informações presentes no SIM. No geral, os municípios apresentam dificuldades em relação à alimentação adequada dos sistemas de informação, portanto, a avaliação da qualidade dos dados sobre mortalidade, por meio da avaliação dos óbitos por causa mal definidas e completitude dos campos do SIM constituem fontes de informação privilegiadas para estudo e o monitoramento.

Diante desse contexto o estudo tem como objetivo comparar a qualidade da informação do Sistema de Informação sobre Mortalidade nos municípios que compõe a VI Região de Saúde de Pernambuco usando como referência o ano 2013.

\section{METODOLOGIA}

O Estado de Pernambuco é organizado em quatro (4) Macrorregiões, 12 Regiões e 11 Microrregiões de Saúde. A VI Região de Saúde de Pernambuco, com sede em Arcoverde, compõe a Macrorregião de Saúde 3 Sertão e é formada por treze municípios com população estimada pelo Instituto Brasileiro de Geografia e Estatística (IBGE) para o ano de 2013 de 404.689 habitantes e área territorial de $13.706,1 \mathrm{~km}^{2}$. O corte populacional definido para essa região está acima daquele preconizado pela literatura como escala mínima de 100 a 150.000 habitantes para o desenho demográfico de uma região de saúde ${ }^{13}$.

Foi realizado um estudo comparativo, de corte transversal e abordagem quantitativa, que utilizou como fonte de dados secundários - Sistema de Informação sobre Mortalidade (SIM) disponível no endereço eletrônico do Departamento de Informática do SUS (DATASUS). Foram considerados os registros de óbitos dos municípios que compõem a VI Região de Saúde de Pernambuco referente ao ano 2013.

A fim de descrever o índice de mortalidade por todas as causas nos municípios que compõem a VI Região de Saúde de Pernambuco em Arcoverde no ano 2013 foram calculados os Coeficientes Geral de Mortalidade (CGM). Os dados sobre estimativas populacionais foram obtidos no Instituto Brasileiro de Geografia e Estatística (IBGE).

Para verificar a qualidade do sistema de informação observou-se o comportamento do grau de preenchimento das variáveis relativas à completitude dos dados e as causas básicas de óbito. Os dados foram tabulados e em seguida organizados e distribuídos para a VI Regional de Saúde e por municípios em tabelas criadas no programa Microsoft Office Excel®. Optou-se por usar os óbitos por 
município de ocorrência uma vez que as DO são preenchidas no local onde ocorre o óbito.

No sítio do DATASUS estão disponíveis as tabulações sobre completitude de informações nomeadas "principais campos do SIM" que incluem as variáveis raça/cor, estado civil, escolaridade e ocupação, além de disponibilizar as causas básicas de óbito mal definidas de acordo com a Décima Revisão da Classificação Internacional de Doenças (CID-10).

Foi atribuído um escore para cada variável de acordo com a sua incompletude, seguindo a metodologia proposta por Romero e Cunha ${ }^{14}$ : excelente para variáveis com menos de $5 \%$ de preenchimento incompleto; bom para variáveis com $5 \%$ e menos que $10 \%$; regular para variáveis com $10 \%$ a menos que $20 \%$; ruim para variáveis com $20 \%$ e menos que $50 \%$ e muito ruim para variáveis com $50 \%$ ou mais.

Especificamente sobre as causas básicas de óbito, utilizou-se como forma de analisar a qualidade da informação do sistema a proporção de óbitos classificados como causas mal definidas de acordo com o CID10 , foram calculados ainda os coeficientes de mortalidade por causas mal definidas.

Para esse estudo foi dispensado à submissão e aprovação do Comitê de Ética em Pesquisa, uma vez que utilizou dados secundários e de domínio público, não havendo identificação dos indivíduos.

\section{RESULTADOS}

A análise do SIM, no ano de 2013, no Estado de Pernambuco (PE), permitiu identificar a ocorrência de 1.635 óbitos na VI
Regional de Saúde - Arcoverde, o que corresponde a um Coeficiente Geral de Mortalidade (CGM) de 4,0 óbitos para 1000 pessoas. Dentre os municípios da VI Regional, o município de Arcoverde apresentou a maior taxa de mortalidade geral 9,1 , no outro extremo os municípios de Tupanatinga $(2,9)$, Manari $(2,7)$, Tacaratu $(2,6)$ e Inajá $(2,5)$ com os menores CGM (Quadro 1).

O Quadro 1 traz os números absoluto e percentual do grau de não preenchimento das variáveis dos blocos da declaração de óbito relacionados com raça/cor, estado civil, escolaridade e ocupação. Para a variável "raça/cor" a variabilidade de dados não preenchidos de informações das DO se manteve entre $0 \%$ a $10,7 \%$, onde os municípios de Inajá e Sertânia preencheram 100\% deste campo em suas declarações de óbitos, por outro lado os municípios de Arcoverde e Petrolândia apresentaram os piores índices de preenchimento dessa variável nas declarações de óbitos, com percentuais próximos a $11 \%$ nos dois municípios. A média para os municípios dessa regional foi de $8,2 \%$ o que confere um padrão de qualidade bom, segundo Romero e Cunha ${ }^{14}$.

A variável "estado civil" chega a ter parâmetros entre $2,27 \%$ a $24,53 \%$ de não completitude, com média entre os municípios de $14,1 \%$ se colocando no patamar de qualidade regular em relação a essa informação. Fazendo a comparação entre os municípios, observa-se que os Municípios de Pedra, Tacaratu e Venturosa, apresentaram índices abaixo de $5 \%$ de grau de não preenchimento, sendo esses municípios classificados pelo escore de Romero e Cunha ${ }^{14}$ como excelente. O Município de Manari, apresentou o pior percentual 


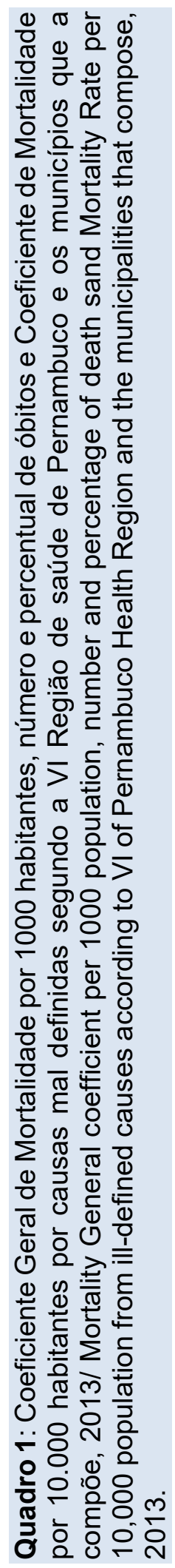

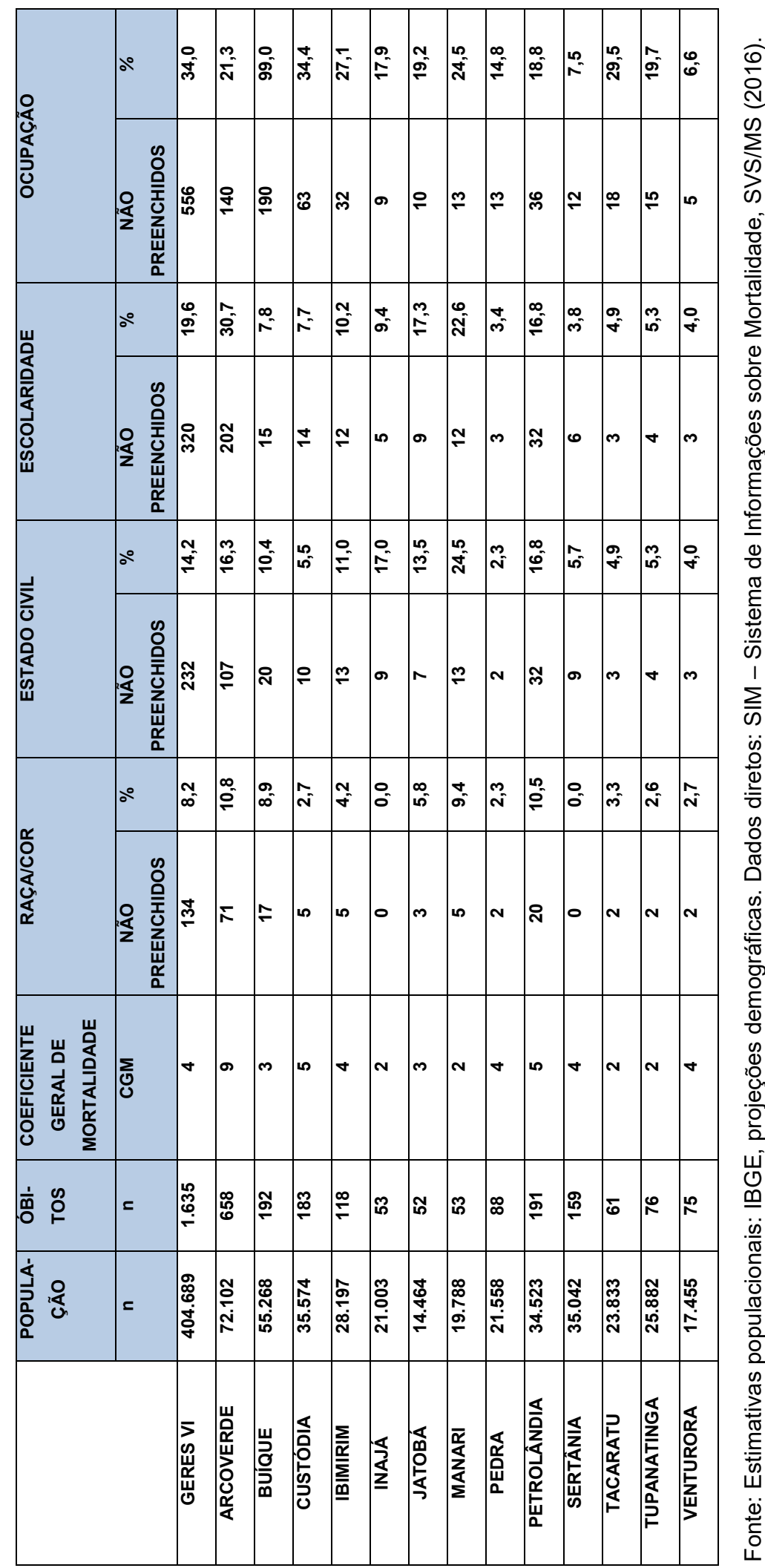


de grau de não preenchimento, chegando a $24,5 \%$, o que classifica o município como ruim pelo escore acima mencionado.

Em relação à variável "escolaridade", a mesma se apresenta com qualidade regular para o ano de 2013, com média de 19,5\% para os Municípios da VI Regional de Saúde. Os Municípios de Pedra, Sertânia, Tacaratu e Venturosa apresentaram as menores taxas de não preenchimento abaixo de $5 \%$, onde esses municípios foram classificados como excelente, dentro da faixa de escore comparativo. No entanto, os Municípios de Arcoverde e Manari tiveram as maiores taxas de não preenchimento, $30,7 \%$ e $22,6 \%$ respectivamente, sendo os mesmos classificados como ruim.

A variável "ocupação" apresenta uma média de $34 \%$ de não preenchimento, se colocando no patamar ruim, porém Municípios como Sertânia e Venturosa apresentaram grau de não preenchimento na faixa de $7,5 \%$ e $6,6 \%$ respectivamente, o que corresponde como bom, porém o Município de Buíque apresentou o pior parâmetro chegando a 98,6\% o que torna o município como muito ruim, segundo o escore de Romero e Cunha ${ }^{14}$. No geral os Municípios de Inajá, Pedra, Sertânia e Venturosa apresentaram melhor qualidade de preenchimento nas variáveis do SIM, com índices mais elevados de completitude dos dados.

Com relação aos óbitos por causas mal definidas, seu registro reflete a qualidade do dado no SIM de uma localidade. Quanto maior a sua proporção, pior a qualidade da informação, prejudicando a análise e conhecimento da realidade ${ }^{15-16}$. O quadro 2 apresenta os coeficientes de mortalidade por 10.000 habitantes e o percentual dos óbitos por causas mal definidas em relação as demais causas de óbito no ano de 2013.

Verificou-se que o município que obteve o maior percentual foi Buíque, apresentando $52,08 \%$ do total dos óbitos por causas mal definidas, seguido por Tupanatinga e Sertânia com $32,89 \%$ e $28,93 \%$, respectivamente. Os municípios que apresentaram os menores percentuais para esse indicador foram $\mathrm{Pe}$ trolândia (3,66\%), Inajá $(5,66 \%)$ e Venturosa $(6,67 \%)$.

\section{DISCUSSÃO}

O comportamento do não preenchimento das variáveis relativas à raça/cor, estado civil, escolaridade e ocupação na VI região de saúde pode ser observado no Quadro 1. A variável "raça/cor" foi introduzida no SIM em 1995, mas somente a partir do ano de 2000 o Ministério da Saúde passou a trabalhar este dado com o intuito de melhorar as estatísticas vitais, podendo investigar dessa forma diferenças entre brancos, negros e pardos.

Nos municípios avaliados observou-se variação no padrão de qualidade de preenchimento desta variável, o que evidencia a existência de limitações para a classificação da raça/cor em recém-nascidos e em óbitos, pois o procedimento mais adequado e amplamente recomendado é o da autoclassificação. Braz et al. ${ }^{17}$ ao avaliarem a completitude da variável raça/cor em diferentes SIS observaram melhoria no preenchimento dessa variável em todos os sistemas, sem, contudo, atingir os percentuais considerados adequados (acima de 90\%) de preenchimento na maioria deles. Sobre o SIM os autores observaram um incremento progressivo a partir de 1997 até 


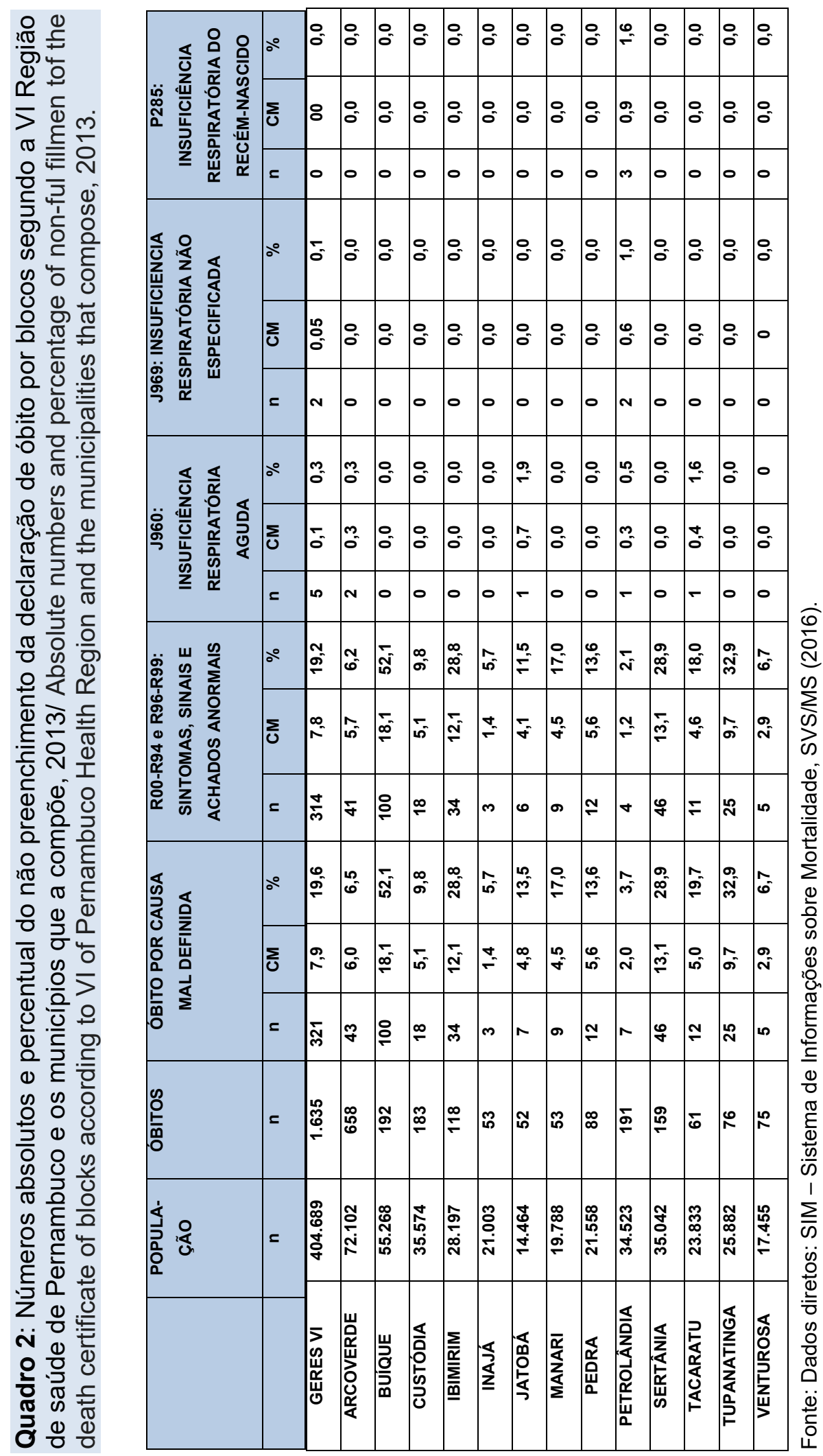


alcançar um percentual maior que $90 \%$ em 2004, mantendo-se assim até 2011.

Quanto à variável "estado civil", houve grande variabilidade, de ruim a excelente, entre os municípios da VI Regional, sendo os maiores índices de não completitude nos Municípios de Manari, Inajá e Petrolândia, respectivamente. Na prática, observa-se que muito desses dados, tanto as variáveis raça/ cor quanto estado civil, são preenchidos pelo pessoal do administrativo das unidades notificadoras, onde o desconhecimento da importância destas variáveis resulta no não preenchimento das mesmas. Apesar de não serem variáveis obrigatórias, ressalta-se a importância de garantir um bom preenchimento de todos os campos da DO, cuja responsabilidade é do profissional médico ${ }^{18}$.

Na variável grau de escolaridade, observa-se que não existe uma homogeneidade intrarregional no grau de completude com índices acima dos 15\%, chegando a 30,7\% no Município de Arcoverde. Poucos são os estudos que se baseiam nessa variável, pois a sua não completitude é muita elevada em todo território brasileiro, chegando a ser preocupante visto que essa variável é uma das mais relevantes em estudos sobre a desigualdade social.

A variável "ocupação" apresentou uma das maiores taxas de não preenchimento entre todos os municípios da VI Regional, chegando a níveis percentuais de $99 \%$ de incompletude. A ocupação é um dado de suma importância, pois reflete direta ou indiretamente o nível socioeconômico da população diagnosticada. No documento "A construção da Política de Informação e Informática em Saúde do Sistema Único de Saúde", de
2003, está muito claro que as características sociodemográficas, tais como idade, gênero, raça/etnia, escolaridade, ocupação e classe social poderão ser utilizadas para a realização de estudos que objetivem a redução das desigualdades em saúde e a ampliação do acesso da população a serviços de qualidade, oportunos e humanizados ${ }^{19}$.

A proporção de "causas mal definidas" tem sido utilizada para avaliar a qualidade dos dados de mortalidade por causas na DO, quanto maior a proporção de óbitos por causas mal definidas em uma distribuição, menor a exatidão dessas estatísticas ${ }^{20}$. As causas mal definidas estão no Capítulo XVIII - Sintomas, sinais e achados anormais de exames clínicos e de laboratório, não classificados em outra parte da Classificação Internacional de Doenças (CID-10) e nela estão inclusos os casos com assistência médica onde não foi possível determinar a causa básica do óbito, e aqueles sem assistência médica ${ }^{20}$.

O não preenchimento dessas variáveis tem sido um sério obstáculo ao conhecimento preciso de importantes indicadores epidemiológicos o que tem levado a prejuízos na produção de dados fidedignos. Assim, a existência de dados confiáveis é condição essencial para a elaboração do diagnóstico, planejamento adequado e para a programação de ações efetivas. Avaliar os sistemas de informação é imprescindível, pois são instrumentos para o diagnóstico situacional, podendo caracterizar populações sob riscos, planejar estratégias conforme as especificidades de cada grupo populacional ${ }^{21}$.

Em Pernambuco, um estudo sobre a completitude dos dados do SIM foi desenvolvido a fim de observar a qualidade do sistema de 
informação sobre mortalidade. Observou-se a heterogeneidade das informações nas diversas regiões de saúde de Pernambuco, ocorrendo melhoria na qualidade das informações entre os anos de 2000 - 2002 a 2008 - 2010 em todo estado ${ }^{18}$.

\section{CONSIDERAÇÕES FINAIS}

É importante reconhecer que o SIM vem melhorando acentuadamente, tanto no que se refere a sua cobertura quanto à qualidade de seus dados. Diante dos dados avaliados e da literatura utilizada foi observado que a qualidade do Sistema ainda é um desafio e que muito pode ser feito a fim de aperfeiçoar as informações sobre mortalidade, por meio de métodos simples e possíveis de serem conduzidos.

A incompletude das variáveis dos óbitos pode influenciar nos resultados encontrados, mascarar ou distorcer informações importantes sobre mortalidade, dificultando a prevenção de mortes evitáveis. Sendo um sistema de abrangência nacional, o SIM é uma fonte confiável dos registros de óbitos no Brasil, sendo os profissionais que trabalham com a DO tanto no seu preenchimento e/ou processamento, os responsáveis pela qualidade dessas informações.

Observou-se grande variabilidade na qualidade das informações sobre mortalidade entre os municípios da VI Região de Saúde. A incompletude dos campos do SIM e o elevado índice de mortes por causas mal definidas refletem a qualidade do preenchimento e processamento das declarações de óbito e, consequentemente, a qualidade do Sistema de informação.

Vale ressaltar a importância do envolvimento dos gestores municipais e estaduais, além de sensibilizá-los quanto à relevância da qualidade dos dados sobre mortalidade, pois esses indicadores demonstram uma melhoria da cobertura e da qualidade das informações, para assim, possibilitar as discussões sobre desigualdades em saúde no país.

\section{REFERÊNCIAS}

1. Machado CB. Avaliação da qualidade do sistema de informação sobre mortalidade no estado do Ceará. $162 f$. Dissertação (Mestrado em Saúde Pública) - Universidade Estadual do Ceará. Fortaleza, 2007.

2. Brasil. Ministério da Saúde. Lei n ${ }^{\circ} 8.080$ de 19 de Setembro de 1990. Dispõe sobre as condições para a promoção, proteção e recuperação da saúde, a organização e o funcionamento dos serviços correspondentes e dá outras providências. Brasília, 1990.

3. Brasil. Ministério da Saúde. Organização Pan-Americana da Saúde. Fundação Oswaldo Cruz. A experiência brasileira em sistemas de informação em saúde. Brasília,2009.

4. Drummond JRM et al. Avaliação da qualidade das informações de mortalidade por acidentes não especificados e eventos com intenção indeterminada. Revista de Saúde Pública, 1999; 33(3):273-280.
5. Matos SG, Proietti FA, Barata RCB. Confiabilidade da informação sobre mortalidade por violência em Belo Horizonte, MG. Revista de Saúde Pública, 2007; 41(1):76-84.

6. Oliveira PPV et al. Confiabilidade da causa básica de óbito por câncer entre Sistema de Informações sobre Mortalidade do Brasil e Registro de Câncer de Base Populacional de Goiânia, Goiás, Brasil. Cad. Saúde Pública, Rio de Janeiro, 2014;30(2):296-304.

7. Brasil. Ministério da Saúde. Manual de procedimentos do Sistema de Informações sobre Mortalidade. Brasília,2001.

8. Baldijão MFA. Sistemas de informação em saúde. São Paulo Perspect, 1992; 6(4):21-28.

9. Brasil. Ministério da Saúde. Conselho Federal de Medicina. Centro Brasileiro de Classificação de Doenças. A declaração de óbito: documentonecessário e importante. Brasília,2009. 
10. Correia LOS, Padilha MB, Vasconcelos SML. Métodos para avaliar a completitude dos dados dos sistemas de informação em saúde do Brasil: uma revisão sistemática. Ciência \& Saúde Coletiva, 2014; 19(11):4467-4478.

11. Felix JD et al. Avaliação da completude das variáveis epidemiológicas do Sistema de Informação sobre Mortalidade em mulheres com óbitos por câncer de mama na Região Sudeste - Brasil (1998 a 2007). Ciência \& Saúde Coletiva, 2012; 17(4):945-953.

12. Pereira CCB et al. Avaliação da implantação do Sistema delnformações sobre Nascidos Vivos (Sinasc) em Pernambuco. Rev. Bras. Saúde Matern. Infant., 2013; 13(1):39-49.

13. Aletras $V$, Jones $A$, Sheldon TA. Economies of scale and scope. In: Ferguson B, Sheldon T.A, Posnett J. - Concentration and choice in health care. London, Financial Times Healthcare,1997.

14. Romero DE, Cunha, CB. Avaliação da qualidade das variáveis sócio- econômicas e demográficas dos óbitos de crianças menores de um ano registrados no Sistema de Informações sobre Mortalidade do Brasil (1996/2001). Cadernos de Saúde Pública, 2006; 22(3):673-684.

15. Costa MR, Marcopito LF. Mortalidade por causas mal definidas, Brasil, 1979- 2002, e um modelo preditivo para a idade. Cadernos de Saúde Pública,2008; 24(5):1001-1012.

16. Veras AP. Esclarecimento de óbitos por causas mal definidas, registradas no Hospital do Pronto Socorro Municipal Dr. Humberto Maradei Pereira, município de Belém do Pará, no ano de 2008. 48f. Dissertação (Mestrado em Saúde Pública) - Escola Nacional de Saúde Pública Sergio Arouca - Fundação Oswaldo Cruz. Belém,2011.
17. Braz RM et al; Avaliação da completude da variável raça/ cor nos sistemas nacionais de informação em saúde para aferição da equidade étnico-racial em indicadores usados pelo Índice de Desempenho do Sistema Único de Saúde. Saúde em Debate. Rio de Janeiro, 37(99):554-562, out/ dez2013.

18. Melo GBT, Valogueiro S. Incompletude dos registros de óbitos por causas externas no Sistema de Informações sobre Mortalidade em Pernambuco, Brasil, 2000-2002 e 2008-2010*. Epidemiol. Serv. Saúde, Brasília, 2015; 24(4):651-660.

19. Brasil. Ministério da Saúde. Secretaria Executiva. Departamento de Informação e Informática do SUS [homepage na Internet]. A construção da política nacional de informação e informática em saúde: proposta versão 2.0: (inclui deliberações da 12. ${ }^{a}$ Conferência Nacional de Saúde). Brasília: Ministério da Saúde; 2004.

20. Laurenti R, Mello Jorge MHP, Gotlieb SLD. A confiabilidade dos dados de mortalidade e morbidade por doenças crônicas não-transmissíveis. Ciência \& Saúde Coletiva, 2204; 9(4):909-920.

21. Zillmer JGV et al. Avaliação da completude das informações do Hiperdia em uma unidade básica do sul do Brasil. Revista Gaúcha de Enfermagem, 2010; 31(2):240-246.

\section{CORRESPONDÊNCIA}

Jonnathan Anderson da Silva Santos

Rua Santa Inês, 158 - Prado. CEP: 55642-200

Gravatá - Pernambuco

E-mail: jonnathananderson@hotmail.com 